\title{
On the Speed of Convergence of the Nearest Integer Continued Fraction
}

\author{
By H. Jager
}

\begin{abstract}
Let $p_{n} / q_{n}$ and $A_{n} / B_{n}$ denote the convergents of, respectively, the regular and the nearest integer continued fraction expansion of the irrational number $x$. There exists a function $k(n)$ such that $A_{n} / B_{n}=p_{k(n)} / q_{k(n)}$. Adams proved that for almost all $x$ one has $\lim k(n) / n=\log 2 / \log G, G=\frac{1}{2}(1+\sqrt{5})$. Here we present a shorter proof of this result, based on a simple expression for $k(n)$ and the ergodicity of the shift operator, connected with the nearest integer continued fraction.
\end{abstract}

Every real irrational number $x$ has a unique representation as a regular continued fraction:

$$
x=a_{0}+\frac{1}{\mid a_{1}}+\frac{1}{\mid a_{2}}+\cdots, \quad a_{n} \in \mathbf{Z}, n \geqslant 0 ; \quad a_{n} \geqslant 1, n \geqslant 1 .
$$

One has $a_{0}=0$ if and only if $x \in(0,1)$. For such an $x$ define

$$
T x:=\frac{1}{\mid a_{2}}+\frac{1}{\mid a_{3}}+\cdots .
$$

A central result in the metric theory of the regular continued fraction states that the operator $T$, working on the space $(0,1) \backslash \mathbf{Q}$, is ergodic if this space is provided with Gauss's measure $\mu$, which is defined by

$$
\mu(E):=\frac{1}{\log 2} \int_{E} \frac{d t}{1+t},
$$

see, e.g., [2, Section 4].

The algorithm for calculating the partial quotients $a_{n}$ in (1) relies heavily on the integer function, since

$$
a_{n}=\left\lfloor\frac{1}{T^{n-1} x^{\prime}}\right\rfloor, \quad x^{\prime}=x-\lfloor x\rfloor, n \geqslant 1 .
$$

If one replaces the integer function in this algorithm by the nearest integer function, one is led to the following half regular continued fraction expansion for a real irrational number $x$ :

$$
\begin{aligned}
x=b_{0}+\frac{\varepsilon_{1}}{\mid b_{1}}+\frac{\varepsilon_{2} \mid}{\mid b_{2}}+\cdots, \quad b_{n} \in \mathbf{Z}, n \geqslant 0 ; \quad b_{n} \geqslant 2, n \geqslant 1 & \\
\varepsilon_{n} & \in\{-1,1\}, n \geqslant 1 ; \quad b_{n}+\varepsilon_{n+1} \geqslant 2, n \geqslant 1 .
\end{aligned}
$$

Received October 21, 1981.

1980 Mathematics Subject Classification. Primary 10A32, 10F20, 28D05, 47A35.

Key words and phrases. Nearest integer continued fraction, individual ergodic theorem. 
This expansion is also unique, see [3, Section 38]. Here $b_{0}=0$ if and only if $x \in\left(-\frac{1}{2}, \frac{1}{2}\right)$, and for such an $x$ the $\varepsilon_{1}$ is the signature of $x$. Let $S$ be the operator working on the space $\left(-\frac{1}{2}, \frac{1}{2}\right) \backslash \mathbf{Q}$, defined by

$$
S x=\frac{\varepsilon_{2} \mid}{\mid b_{2}}+\frac{\varepsilon_{3} \mid}{\mid b_{3}}+\cdots .
$$

Recently, Rieger [4] proved that $S$ is ergodic if the space is provided with the measure $\nu$ defined as follows

$$
\nu(E):=\frac{1}{\log G} \int_{E} \rho(t) d t
$$

where

$$
G=\frac{1}{2}(1+\sqrt{5}) \text { and } \rho(t)= \begin{cases}(G+t)^{-1} & \text { for } 0<t<\frac{1}{2} \\ (1+G+t)^{-1} & \text { for }-\frac{1}{2}<t<0\end{cases}
$$

see also [5].

Denote by $p_{n} / q_{n}$ the convergents of (1) and by $A_{n} / B_{n}$ those of (2), $n \geqslant 0$. For every $n \geqslant 0$ there exists a unique $k(n)$ such that $A_{n} / B_{n}=p_{k(n)} / q_{k(n)}$, and then one has

$$
\frac{A_{n+1}}{B_{n+1}}=\frac{p_{k(n)+1}}{q_{k(n)+1}} \quad \text { or } \quad \frac{A_{n+1}}{B_{n+1}}=\frac{p_{k(n)+2}}{q_{k(n)+2}},
$$

the latter case occurring if and only if $a_{k(n)+2}=1$. For a simple proof of these facts we refer to [6, Theorem 2]. This criterion was used by Adams [1] to express $k(n)$ in terms of the occurrence of strings of l's in the sequence $a_{n}, n \geqslant 1$, of partial quotients of (1). He then applied in an ingenious way the individual ergodic theorem with the operator $T$ and obtained the following remarkable result:

THEOREM (ADAMS). For almost all $x$ one has

$$
\lim _{n \rightarrow \infty} \frac{k(n)}{n}=\frac{\log 2}{\log G}=1.4404 \ldots .
$$

The purpose of this note is to give a shorter proof of this theorem, based on a simpler expression for $k(n)$ and on the ergodicity of the operator $S$.

LEMMA. Let $s(n)$ denote the number of negative elements in the sequence $\varepsilon_{1}, \varepsilon_{2}, \ldots, \varepsilon_{n}$. Then $k(n)=n+s(n+1)$.

Proof. It suffices to show that the latter case in (3) occurs if and only if $\varepsilon_{n+2}=-1$. We recall the well-known alternating character of the sequence of convergents of a regular continued fraction

$$
\frac{p_{0}}{q_{0}}<\frac{p_{2}}{q_{2}}<\cdots<x<\cdots<\frac{p_{3}}{q_{3}}<\frac{p_{1}}{q_{1}}
$$

and the relation

$$
A_{n} B_{n+1}-A_{n+1} B_{n}=(-1)^{n+1} \varepsilon_{1} \varepsilon_{2} \cdots \varepsilon_{n+1},
$$

see [3, p. 14]. Suppose that the latter case of (3) occurs together with $\varepsilon_{n+2}=1$. Then, if $k$ is even, we have in view of (4)

$$
\frac{A_{n}}{B_{n}}=\frac{p_{k}}{q_{k}}<\frac{p_{k+2}}{q_{k+2}}=\frac{A_{n+1}}{B_{n+1}} \quad \text { or } \quad A_{n} B_{n+1}-A_{n+1} B_{n}=-1 .
$$


Then (5) and $\varepsilon_{n+2}=1$ would give

$$
A_{n+1} B_{n+2}-A_{n+2} B_{n+1}=1,
$$

i.e., for some $l>k+2$ we would have

$$
\frac{A_{n+1}}{B_{n+1}}=\frac{p_{k+2}}{q_{k+2}}>\frac{p_{l}}{q_{l}}=\frac{A_{n+2}}{B_{n+2}},
$$

which contradicts (4). The case that $k$ is odd is treated similarly. In the same way one shows that the first case of (3) is incompatible with $\varepsilon_{n+2}=-1$. This finishes the proof of the lemma.

To prove the theorem we determine $\lim _{n \rightarrow \infty} s(n) / n$. Let $f$ denote the characteristic function of the interval $\left(-\frac{1}{2}, 0\right)$. Then

$$
s(n)=\sum_{k=0}^{n-1} f\left(S^{k} x^{\prime}\right), \quad x^{\prime}:=x-b_{0} .
$$

From the above quoted result of Rieger and the individual ergodic theorem, we find

$$
\lim _{n \rightarrow \infty} \frac{s(n)}{n}=\frac{1}{\log G} \int_{-1 / 2}^{0} \frac{d t}{1+G+t}=-1+\frac{\log 2}{\log G},
$$

see also [5, Corollary 3]. From this and the lemma, Adams' theorem follows at once.

One of the beautiful results in the metric theory of the regular continued fraction is Paul Lévy's theorem which states that for almost all $x$

$$
\lim _{n \rightarrow \infty} \frac{1}{n} \log q_{n}=\frac{\pi^{2}}{12 \log 2} .
$$

Since

$$
\frac{1}{n} \log B_{n}=\frac{k(n)}{n} \frac{1}{k(n)} \log q_{k(n)},
$$

Adams' theorem immediately transforms Lévy's theorem into the corresponding one for the nearest integer continued fraction: For almost all $x$ one has

$$
\lim _{n \rightarrow \infty} \frac{1}{n} \log B_{n}=K
$$

with

$$
K=\frac{\pi^{2}}{12 \log G}
$$

Both Rieger in [4] and Rockett in [5] find $K$ in the form

$$
K=-\int_{-1 / 2}^{1 / 2} \rho(t) \log |t| d t
$$

Rockett leaves this integral for what it is, whereas Rieger has to use a functional equation for the dilogarithm to prove that its value is given by (6). 
1. William W. ADAmS, “On a relationship between the convergents of the nearest integer and regular continued fractions," Math. Comp., v. 33, 1979, pp. 1321-1331.

2. P. BillingsleY, Ergodic Theory and Information, Wiley, New York, 1965.

3. O. Perron, Die Lehre von den Kettenbrüchen, Vol. I, 3rd ed., Teubner, Leipzig, 1954.

4. G. J. RIEGER, “Mischung und Ergodizität bei Kettenbrüchen nach nächsten Ganzen," J. Reine Angew. Math., v. 310, 1979, pp. 171-181.

5. A. M. Rockett, "The metrical theory of continued fractions to the nearer integer," Acta Arith., v. 38, 1980, pp. 97-103.

6. H. G. Williams, "Some results concerning the nearest integer continued fraction expansion of $\sqrt{D}$," J. Reine Angew. Math., v. 315, 1980, pp. 1-15. 